\title{
ISOTOPENPRAXIS
}

herausgegeben von J. Mühlenpfordt, Berlin

unter Mitwirkung von M. F. Abdel-Wahab, Khartum · O. Charamza, Olomouc . Z. Dienstbier, Praha · L. Herforth, Dresden · V. K. Iya, Bombay · W. Kodjukow, Moskau · M. Melnikowa, Leningrad . V. Mercea, Cluj · R. Münze, Rossendorf - S. Muždeka, Beograd · M. Radwan, Swierk · A. I. Schatenstein, Moskau • H. R. Schütte, Halle . P. Têtênyi, Budapest . K. Unger, Quedlinburg . S. Varga, Bratislava · G. Vormum, Berlin

REDAKTIONSAUSSCHUSS : H.-J. Altenbrunn, Berlin · E. Becker, Magdeburg · W. Dietzsch, Dresden · D. Herrmann, Berlin . J. Holzhey, Freiberg · H. Hübner, Leipzig · K. Irmer, Dresden · P. Krumbiegel, Leipzig · P. Mühl, Dresden · S. Niese, Rossendorf · R. Otto, Leipzig - U: Prösch; Berlin . G. Reinhard, Dresden · H. Scheel, Berlin · K. Wagner, Berlin

REDAKTIONSSEKRETÄR: Frau H. Neumann, Berlin

\section{An unsere Autoren}

Die Zeitschrift veröffentlicht Beiträge zu folgenden Themen: Kontrolle und Analyse radioaktiver und stabiler Isotope, Anwendung radioaktiver und stabiler Isotope in allen Gebieten der Forschung und Technik, der Industrie, landirtschatt und Medizin, Herstelling radioaktiver and stabller Isotope sowie von markierten Verbindungen, kernelektronische Gerate für die Isotopenanwendung (Dickenmesser, Dichtemesser u. a.) Detektoren für isotopentechniohe Arbeiten und den Strahlenschutz in Iotopenlaboratorien, MeBtechnik und fir dis rate lenchemie Radioc

\section{Aufnahme von Manuskripten}

Zur Veröffentlichung angenommen werden nur druckreife, noch nicht anderweitig publizierte Beiträge in deutscher, englischer oder russischer Sprache wum Thementereich der Zeitschrift und zwar

Originalarbeiten

Kurze Mitteilungen

Aus der Praxis

Zusanmenfassonde Berichte

Tagungsherichte

Informationen

Sind der Beitrag oder Teile daraus bereits veröffentlicht oder ist eine anderweitige Veröffentlichung vorgesehen, so muß dies der Redaktion mit genauer Angabe der Zweitveröffentlichung mitgeteilt werden. Mit der Annahme de Manuskriptes behkilt sich der Verlag das Recht der Vervielfältigung, Verbreitung und Ubersetzung innerhalh der gesetzlichen Schutzfrist vor. Auszugsweiser Nachdruck oder photomechanische u. a. Wiedergabe sind an eine schriftiche Genehmigung des Verlages gebunden. Manuskripte, Zeichnungen bis zum Zeitpunkt der Veröffentlichung mitgeteilten Wunsch des Autors zurückgesandt.

Bei der unverlangten Einreichung von Manuskripten wird empfohlen, eine Befürwortung eines der Herausseber oder RedaktionsausschuBmitglieder beizufügen.

Manuskripte sind einzureichen bei der Redaktion, 1086 Berlin, Leipziger Str. 3 -4.

2. Form der Manuskripte

Die Autoren werden gebeten:

2 Manuskripte, eines davon in Original-Maschinenschrift, einseitig und mit 2zeiligem Abstand einzureichen; gut leserliche Photokopien sind zulissig; auf cm 1. Blatt (unter Weglassung der Bezeichnung) en Titel der Arbeit

Vorname-Initialen und Namen säntlicher Antoren

gegebenenfalls die Institution, in der sie tätig sind
eine kurze Zusammenfassung der Arbeit von höchstens 15 Zeilen

Keywords in alphabetischer Reihen folge und

els Fußnute die Anschrift der Institution anzugebon:

wenn moglich, auf gesonderten Blättern Namen, Titel der Arbeit und $\mathrm{Zu}$ wenn moglich, auf gesonderten Blattern Namen, Titel der Arbeit und Zuzufügen;

Literaturhinweise (fortlaufend numeriert), Fußnoten, Tabellen, Abbildungen

(In 2-3tacher Veröffentlichungsgröße), Abbildungsunterschriften (getrennt von den Abbildungen mit Zeichenerklärungen, Quellenangaben, falls erforderlich, und gegebenenfalls mit einem Vermerk far besondere Wünsche bezüglich der AbbildungsgröBe), private und Dienstanschrift der Verfasser mit akademischen Titeln und ausgeschriebenen

Wünsche für den Kolumnentitel (Seitentberschrift) bekanntzugeben;

Absătze durch Einzüge von mindestens 3 Anschlägen kenntlich zu machen; die MaBrinhelten des SI-Systems (,Systeme International") zu verwenden: Korrekturen im Manuskript unmittelbar an der zu korrigierenden Stelle anzubringen;

Formeln deutlich lesbar und möglichst groß einzutragen;

Farbabbildungen und nachträgliche Anderungen oder Ergänzungen in $\mathrm{Ab}$. bildungen zu vermeiden;

hervorzuhebende Textstellen zu unterstreichen, und zwar halbfetten Satz mit einer geraden schwarzen Linie, kursiven Satz (Schrägschrift) mit einer mitauen Wellenlinie (alle Bigennamen). Sperrungen gestrichelt;

besondere Buchstaben durch farbige Unterstreichung $z \mathrm{u}$ kennzeichnen, und zwar Formelzeichen in

Griechisch-Kursiv $(\alpha, \beta, \ldots)$ rot,

Kursiv $(A, B, \ldots)$ blau

halb fetter Kursiv $(\boldsymbol{A}, \boldsymbol{B}, \ldots) 2$ Linien blau (Vektoren),

halb fetter Grotesk (A, B, ...) 2 Linien violett (Tensoren);

bei Literaturangaben auf Vollstándigkeit zu achten. In nachstehender Reihenfolge müssen angegeben sein:

a) bei Zeitschriften: Vorname-Initialen und Namen ss్stlicher Autoren, Titel der Zeitschrift (nach ,Periodica Chimica" oder möglichst wenig gekürzt), Bandnummer (unterstrichen), Jahreszahl (in Klammern), Heftnummer (nu bei Zeitschriften, die jedes Heft mit $\mathrm{S} .1$ beginnen) sowie die Seitenzahl, auf de die zitierte Arbeit beginnt.

b) Bei Bilchern: Initialen der Vornamen und Name des Autors (Herausgeber werden durch den Zusatz ,ed.“ gekennzeichnet), voll ausgeschriebener Titel des Buches in der Originaleprache, evtl. Bandnummer, voll ausgeschriebener Name des Verlages, Nummer der Autlage (außer der ersten), Erschelnungsort(e), Erscheinungsjahr
Kapitelnummer, Seitenzahl $\mathbf{u}$. a.

c) bei Sammelbänden, Tagungsberichten: Ks soll zusatzlich der Titel des zitierten Beitrags angegeben werden. Die Seitenzahl muB unbedingt angegeben werden. Aus dem Zitat muß hervorgehen, ob es sich um einen Tagungssammelband oder um einen noch nicht veröffentlichten Vortrag handelt. Zwischen Tagungsort und Erscheinungsort des entsprechenden Tagungsberichts ist zu unterscheiden.

3. Korrekturen

Der Autor erhält zwei Umbruchabzlige, von denen einer möglichst schnell korrigiert an die Redaktion zurückgereicht werden soll. Auf Wunsch des Autor kann die Korrektur auch von der Redaktion gelesen werden.

Durch nachträgliche Manuskriptănderungen verursachte Korrekturkosten gehen, sofern sie $10 \%$ der Satzkosten übersteigen, zu Lasten des Autors.

Der Autor erhălt 50 Sonderdrucke kostenlos. Weitere Sonderdrucke konnen bis zur Rückgabe der Korrekturabzüge auf Kosten des Autors bef der Redaktion bestellt werden.

\section{Bezugsmöglichkeiten}

Bestellungen sind zu richten

- In der DDR an den Postzeitungsvertrieb unter Angabe der Kundennummer des Bestellenden oder an den AKADEMIE-VERLAG, DDR-1086 Berlin, Leipziger StraBe 3-4, PF-Nr. 1233;

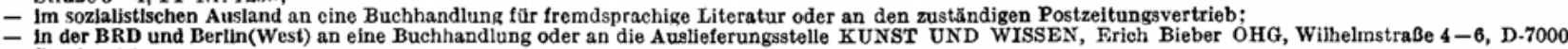
Stuttgart 1;

- In den ubrigen westeuropälschen Ländern an eine Buchhandlung oder an die Auslieferungsstelle KUNST UND WISSEN, Erich Bieber GmbH, Dufourstraße 51, CH-8008 Zürich;

- Im ubrigen Ausland an den Internationalen Buch- und Zeitschriftenhandel; den Buchexport, Volkseigener AuBenhandelsbetrieb der Deutschen Demokratischen Republik, DDR-7010 Leipzig, Postfach 160; oder an den AKADEMIE-VERLAG, DDR-1086 Berlin, Leipziger StraBe 3-4.

Zeitschrift „Isotopenpraxis"

Herausgeber: Im Auftrag des Akademie-Verlages herausgegeben von Prof. Dr. Justus Mühlenpfordt

Verlag: Akademie-Verlag, DDR-1086 Berlin, Leip7iger Straße 3-4, PF-Nr. 1233; Fernruf: 2236221 oder 2236229 ; Telex-Nr.: 114 420;

Bank: Stastsbank der DDR, Berlin, Kto.-Nr.: 6836-26-20712

Verantwortlich für den Inhalt des Heftes: Prof. Dr. H. Hübner, Leipzig

Anschrift der Redaktion (Fernruf Frau H. Schier: 635 1177) der Zeitschrift Isotopenpraxis: Akademie-Verlag, DDR-1086 Berlin, Leipziger Str. $3-4$, PF-Nr.1233 Veróffentlicht unter der Lizenznummer 1527 des Presseamtes beim Vorsitzenden des Ministerrates der Deutschen Demokratischen Republik

Gesamtherstellung: VEB Druckerei "Thomas Müntzer", DDR-5820 Bad Langensalza

Brscheinungsweise: Die Zeitschrift "Isotopenpraxis“ erscheint monatlich. Die 12 Hefte eines Jahrganges bilden einen Band. Bezugspreis je Band 216,- MI zuzuglich Versandspesen. Preis je Heft 18,-

Bestellnummer dieses Heftes: $1079 / 20 / 9$

(c) 1984 by Akademie-Verlag Berlin. Printed in the German Democratic Republic

AN (EDV) 12831 